\title{
Coherence of the Even-Odd Rule with an Effective-Valence Isoelectronicity Rule for Chemical Structural Formulas: Application to Known and Unknown Single-Covalent-Bonded Compounds
}

\author{
Geoffroy Auvert \\ CEA-Leti, Grenoble, France \\ Email: Geoffroy.auvert@grenoble-inp.org
}

Received 5 June 2014; revised 30 June 2014; accepted 25 July 2014

Copyright (C) 2014 by author and Scientific Research Publishing Inc. This work is licensed under the Creative Commons Attribution International License (CC BY). http://creativecommons.org/licenses/by/4.0/ (c) (i)

\begin{abstract}
Ions or molecules are said to be isoelectronic if they are composed of different elements but have the same number of electrons, the same number of covalent bonds and the same structure. This criterion is unfortunately not sufficient to ensure that a chemical structure is a valid chemical compound. In a previous article, a procedure has been described to draw 2D valid structural formulas: the even-odd rule. This rule has been applied first to single-bonded molecules then to single-charged single-bonded ions. It covers hypovalent, hypervalent or classic Lewis' octet compounds. The funding principle of the even-odd rule is that each atom of the compound possesses an outer-shell filled only with pairs of electrons. The application of this rule guarantees validity of any single-covalent-bond chemical structure. In the present paper, this even-odd rule and its electron-pair criterion are checked for coherence with an effective-valence isoelectronic rule using numerous known compounds having single-covalent-bond connections. The test addresses Lewis' octet ions or molecules as well as hypovalent and hypervalent compounds. The article concludes that the even-odd rule and the effective-valence isoelectronicity rule are coherent for known single-covalent-bond chemical compounds.
\end{abstract}

\section{Keywords}

Isoelectronicity, Effective Valence, Molecule, Ion, Even-Odd, Rule, Structural Formula, Covalent Bond 


\section{Introduction}

A chemical structural formula of a compound is a two-dimensional representation procedure initiated about two centuries ago. Before 1811, atoms and molecules were thought to be similar entities. Avogadro [1], using the law of multiple proportions adopted by John Dalton in 1803 [2], introduced the term "molecules" to describe basic components of gases. This conception was argued with until Albert Einstein and Jean Perrin explained the Brownian movement in a liquid by the presence of molecules. Einstein used theory [3] and Perrin experiment [4].

Following the discovery of the electrical properties of liquids, Michael Faraday coined the term "ions" to describe compounds carrying electrical charges [5].

When comparing both, the term "isoelectronicity" [6] is used for ions and molecules having nearly the same structures. With this concept, a compound is said to be isoelectronic if it has the same number of electrons [7] [8]. Unfortunately, this property does not always give a chemically valid structure.

The recently proposed "even-odd" rule [9] [10], introduced as a procedure to draw chemical structural formulas of ions and molecules, has not yet taken into account a possible isoelectronicity link between the represented compounds.

The aim of this paper is to check the coherence of the "even-odd" rule using compounds with a specific type of isoelectronicity named "effective-valence isoelectronicity". Structural formulas of compounds: cations, molecules and anions, following this rule are drawn including numbers required by the even-odd rule. These numbers, associated with each element, are calculated and discussed. The state of scientific knowledge of these compounds is taken into account as well as specific isoelectronic links and resulting even-odd structures.

\section{Even-Odd Rule for Ions and Molecules}

The even-odd rule is a procedure to draw chemical structural formulas of molecules and ions. The structure is composed of one or several atoms of the periodic table.

As a reminder [10], the rule is described below:

- Each atom:

- Is an element with one or several electron shells.

- Possesses an outer-shell filled with one to eight electrons.

- Has a number, also called valence number, of electrons in the outer-shell as indicated in the periodic table.

- Has a valence number of the element giving the highest number of covalent bonds that the element can form.

- A structure meets the criteria below:

- When it is composed of only one atom, it forms no covalent bond.

- When it is constituted of several atoms, each atom forms a single covalent bond with each of its first neighboring atoms. This covalent bond involves two electrons, one from each interconnected atom.

- A covalent bond is represented by one line between both connected atoms.

- An atom may have zero, one or more than one line around it.

- In the 2D structure, each atom is represented by the letters of its element as in the periodic table.

- Two numbers have to be evaluated and written on each side of the atom.

- The left side number and the effective valence number:

- The left side number is the valence number as in the periodic table. It ranges from one for elements like sodium (Na) up to eight for noble gas like Argon (Ar).

- An effective valence number has to be evaluated: For a neutral atom i.e. without charge, it is equal to the valence number; for a negatively charged atom i.e. that possesses an extra-electron, it is the valence number increased by one; for a positively charged atom, it is the valence number decreased by one.

- The right side number of an atom:

- The right side number, the "Lewis number", is equal to the sum of the effective valence number and the number of covalent bonds of the atom. It can also be expressed as the sum of the number of electrons left in the outer-shell and twice the number of covalent bonds.

- The Lewis number must be an even number. This is only possible when the number of bonds and the effective valence number are either both odd or both even.

- The smallest value the Lewis number can take is zero: the atom has lost electrons from the outer-shell so it is empty and the atom has no bonds. 
- The Lewis number can range up to twice the effective valence number: this is twice the maximum number of covalent bonds for this element. This number is charge dependent through the effective valence number.

- If all atoms of a compound have Lewis numbers equal to eight, the compound is compatible with Lewis' octet rule.

- Electron pairs in the outer-shell of an interconnected atom:

- The number of electrons in the outer-shell is calculated by subtracting the effective valence-number and the number of covalent bonds. It is an even number.

- As a consequence, the outer-shell contains electron pairs not involved in any covalent bond.

- This electron-pair number ranges from 0 to 4 whatever the charge of the element.

- When this electron-pair number is 0 , no additional covalent bond can be formed by the element.

The even value of the right side number i.e. the Lewis number, and the even value of the number of electrons in the outer-shell are important keys to the validity of the even-odd rule. With these even values, molecules and ions belong to a group of electron-paired compounds [9] [10].

\section{Effective-Valence Isoelectronicity Rule}

An even-odd compound changes into a valid isoelectronic one when it follows the effective-valence isoelectronicity rule described below:

- The new compound configuration:

- Has the same covalent-bond structure.

- Has at least one different atom.

- The replacing atom has no impact on the outer-shell configuration:

- It builds the same number of single covalent-bonds.

- It has the same effective-valence number, as defined in the even-odd rule.

- It has the same Lewis number i.e. right side number.

- It has the same number of electrons in the outer-shell.

- But its internal configuration is different:

- With a different name from the periodic table.

- It meets one of the following criteria:

- It belongs to the same column of the periodic table (same valence number).

- It belongs to the same line of the periodic table and the valence number is corrected by a charge modification giving the same effective-valence number.

- It is shifted in column and lines of the periodic table and with a charge modification to maintain constant the effective-valence number.

From the first criteria, one example is for $\mathrm{BH} 4(-)$ and $\mathrm{GaH} 4(-)$. The effective-valence isoelectronicity comes from Boron and Gallium. They are in the same column of the periodic table. Only their names are different. All other parameters of the rule are the same. Since both are in the same column of the periodic table, their isoelectronicity link is obvious. Similar cases are therefore not discussed further in this article.

From the second criteria, one example is for $\mathrm{HF}$ and $\mathrm{OH}(-)$. The effective-valence isoelectronicity comes from $\mathrm{O}(-)$ and $\mathrm{F}$. They are on the same line in the periodic table. Both have one covalent-bond, an effective valence of seven, a right side number of 8 and six electrons in the outer-shell.

From the third criteria, one example is for $\mathrm{CH} 4$ and $\mathrm{PH} 4(+)$. The effective-valence isoelectronicity comes from $\mathrm{C}$ and $\mathrm{P}(+)$. They are not on the same line of the periodic table and not in the same column. Both have 4 covalent-bonds, an effective-valence number of 4 , a right-side number of 8 and no electrons in the outer-shell.

\section{Isoelectronicity between Single-Charged Single-Bonded Ions and Neutral Single-Bonded Molecules}

In the following, the coherence of the even-odd rule with the effective-valence isoelectronicity rule is tested for several known compounds, ions or molecules. Due to the large number of these compounds, the scope of the test is deliberately limited: firstly to compounds composed of main group elements; secondly to compounds having a star configuration. A star configuration is structured with a central atom connected to each peripheral atom via a single covalent bond. Peripheral atoms have no other bonds. To build isoelectronic compounds of such structures, it is sufficient to replace the central atom by one of its effective-valence isoelectronic atoms. 
Table 1. Compounds with an effective-valence isoelectronicity. On the same line, compounds are isoelectronic. A group of lines groups compounds with central atoms having the same Lewis number i.e. the right side number calculated with the even-odd rule. Column 1 gives the value of the right side number of the group of isoelectronic compounds and its classical formulas. Column 2 lists cations, column 3 neutral molecules and column 4 anions. Column 5 indicates the number of electrons in the outer-shell of all three isoelectronic compounds. In column 1, when "No" stands in place of a classical formula of a compound, it means that the corresponding compounds is not referred to in any scientific literature, being in fields of physics or chemistry. The left side number (the valence number) of the element is indicated for each central atom and according to the even-odd rule. The right side number i.e. Lewis number, is the sum of the effective-valence number of the atom with the number of its single covalent-bonds. Note: the isoelectronicity in the same periodic column are not reported. (See text with BH4-GaH4).

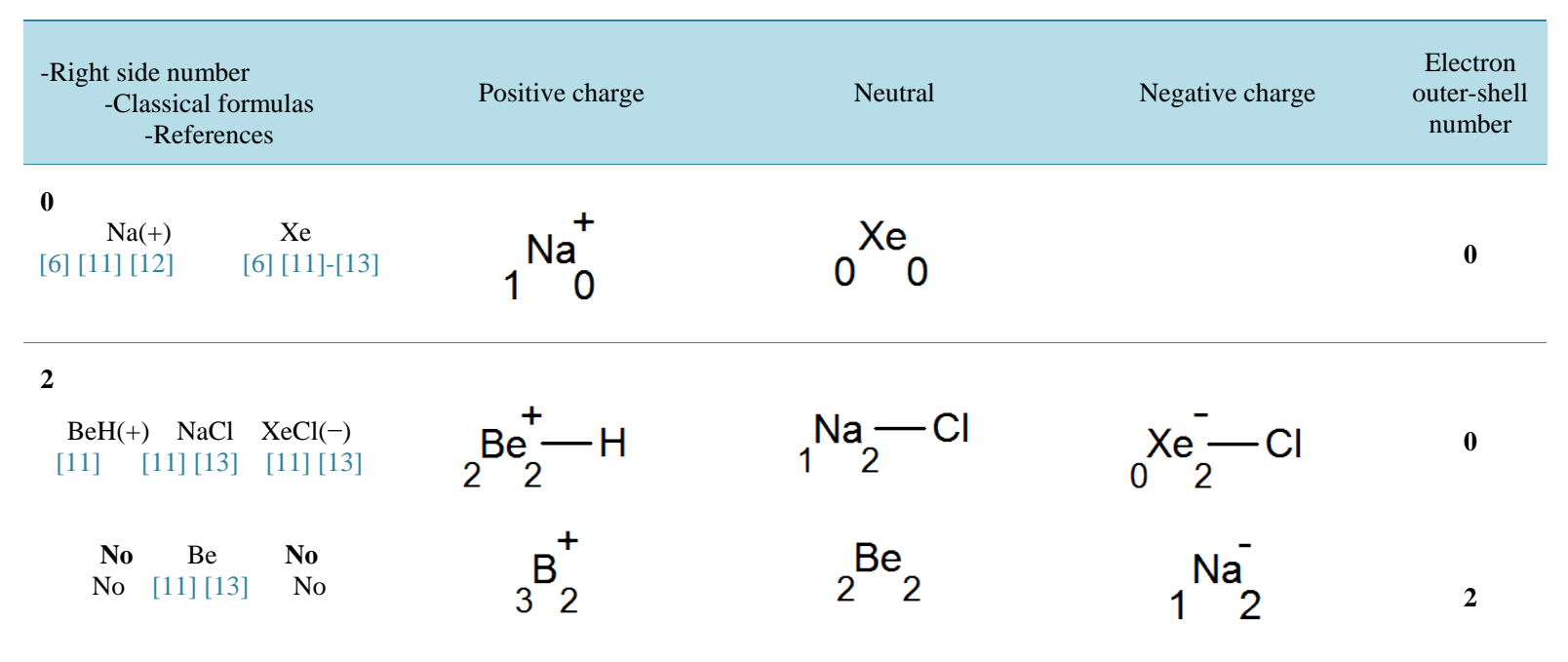

4

$\begin{array}{ccc}\text { BF2(+) } & \text { BeF2 } & \text { No } \\ {[11]} & {[13]} & \text { No } \\ & & \\ \mathrm{SiF}(+) & \text { BH } & \text { BeH(-) } \\ {[11]} & {[11]} & {[11]}\end{array}$

$\begin{array}{ll}\text { No } & \text { No } \\ \text { No }[11][13] \quad \text { No }\end{array}$

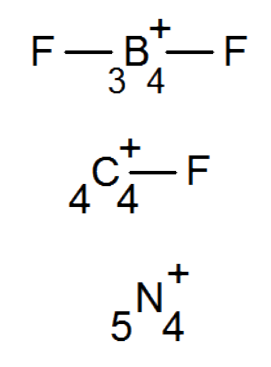

$\mathrm{F}-\mathrm{Be}_{4}-\mathrm{F}$

$3-\mathrm{B}-\mathrm{H}$

${ }_{4}{ }_{4}$ o

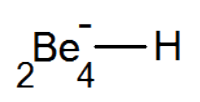

2

\section{$3^{B^{-}}$}

6

CH3(+) BF3 BeF3(-)

[11]-[13] [11]-[13] [11] [12]

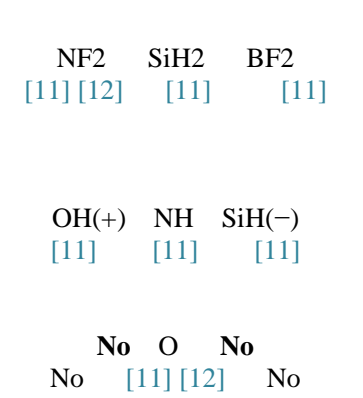

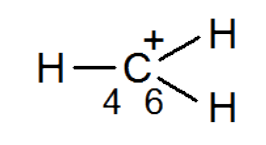

$\mathrm{F}-\mathrm{N}_{6}^{+}-\mathrm{F}$<smiles>[O+][O-]</smiles>

${ }_{7}^{\mathrm{F}_{6}^{+}}$<smiles>[BH3-]</smiles><smiles>[2H][Se](F)(F)F</smiles><smiles>[13CH3][13CH3]</smiles>$$
\mathrm{F}-\mathrm{B}_{6}^{-}-\mathrm{F}
$$<smiles>[13NH2]</smiles>

${ }_{4} \mathrm{Si}_{6}^{-}-\mathrm{H}_{2}$
${ }_{6} \mathrm{O}_{6}$
${ }_{5} \mathrm{~N}_{6}^{-}$ 


\section{Continued}

8

NH4(+) $\quad \mathrm{CH} 4 \quad \mathrm{BH} 4(-)$

[11]-[13] [11]-[13] [11]-[13]

$\mathrm{H} 3 \mathrm{O}(+)$ NH3 $\mathrm{CH} 3(-)$

[11]-[13] [11]-[13] [11]-[13]

$\mathrm{F} 2 \mathrm{Cl}(+) \quad \mathrm{H} 2 \mathrm{O} \quad \mathrm{NF} 2(-)$

[11]-[13] [11]-[13] [11] [12]

$\mathrm{XeF}(+) \quad \mathrm{HCl} \mathrm{OH}(-)$

[6] [13] [11]-[13] [11]-[13]

$\begin{array}{cc}\mathrm{Xe} & \mathrm{F}(-) \\ {[11][13]} & {[6][11][13]}\end{array}$<smiles>[NH3+][SH2+]</smiles><smiles>C</smiles>

$\mathrm{H} \backslash \mathrm{H}_{3}-\mathrm{B}_{8}>\mathrm{H}$
$-\mathrm{H}$<smiles>[NH3+]</smiles>

$\mathrm{H}-\underset{4}{\mathrm{C}}-\mathrm{C}$

$\mathrm{H}-{ }_{6} \mathrm{O}-\mathrm{H}$

$\mathrm{F}-\mathrm{N}_{8}^{-}-\mathrm{F}$

${ }_{7} \mathrm{Cl}-\mathrm{H}$

$6 \mathrm{O}_{8}^{-}-\mathrm{H}$

$$
{ }_{8} \mathrm{Xe} \frac{+}{8} \mathrm{~F}
$$

None

${ }_{7} \mathrm{~F}_{8}^{-}$
0

10

SF5(+) PF5 SiH5(-)

[11] [12] [11]-[13] [11]

$\mathrm{F} \underset{\mathrm{F}>6}{>} \mathrm{S}_{10}^{+} \frac{\mathrm{F}}{\mathrm{F}}$

$\underset{\mathrm{F}>5}{\mathrm{~F}}>\mathrm{P} \frac{\mathrm{P}}{10} \underset{\mathrm{F}}{\mathrm{F}}$

$\underset{\mathrm{H}}{\mathrm{H}}>\frac{\mathrm{Si}}{10} \underset{-}{-}=\frac{\mathrm{H}}{\mathrm{H}}$

0

IF4(+) SF4 PF4(-)

$\mathrm{F}>>1+\mathrm{I}_{10}^{+}<\mathrm{F}$

$F>S>F$

$F>\frac{F}{F} \underset{10}{ }<F$

[6] [12] [13] [11]

$$
\mathrm{F}-\mathrm{Xe}_{8}^{+} \frac{\mathrm{T}}{10 \mathrm{~F}}
$$

$\mathrm{F}-\mathrm{Br} \underset{10}{ }<\mathrm{F}$

$\mathrm{H}-\mathrm{Se}_{6}^{-} \underset{10}{-}-\mathrm{H}$

$\mathrm{XeF3}(+) \quad \mathrm{BrF} 3 \quad \mathrm{SeH} 3(-)$

[12]

None

$F-{ }_{8} e_{10}-F$

$\left|-1 \frac{-}{7}\right|$

6

[11]-[13] $\quad$ [11]-[13]

No

None

None

${ }_{8} \mathrm{Xe}_{10}^{-} \mathrm{F}$

8

12

IF6(+) SeF6 AsF6(-)

[6] [11]-[13] [11]-[13]

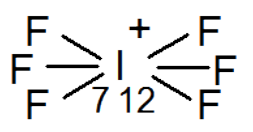

$\underset{F}{F} \underset{F}{F} \operatorname{se} \underset{12}{-} \underset{F}{F}$

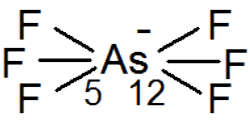

$\mathrm{F}_{\mathrm{F}>\mathrm{T}}^{\mathrm{F}}>\mathrm{F}$

$\underset{F}{\mathrm{~F}}>\frac{\mathrm{s}}{\mathrm{S}} \underset{12}{-}=\mathrm{F}$

$\mathrm{XeF5(+)} \mathrm{IF5} \mathrm{SF5(-)}$

$\underset{\mathrm{F}}{\mathrm{F}}>_{8} \mathrm{Xe} \frac{+}{12} \frac{\mathrm{F}}{\mathrm{F}}^{\mathrm{F}}$

None

$\mathrm{XeF} 4$

$\mathrm{ICl} 4(-)$

[11]

[6] [11]-[13]

No

None

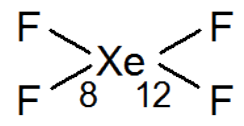

None
$\mathrm{Cl} \backslash->\mathrm{Cl}$
$\mathrm{Cl}>12>\mathrm{Cl}$

$F-X_{8}^{-} \frac{-}{12} F$
0

2 


\section{Continued}

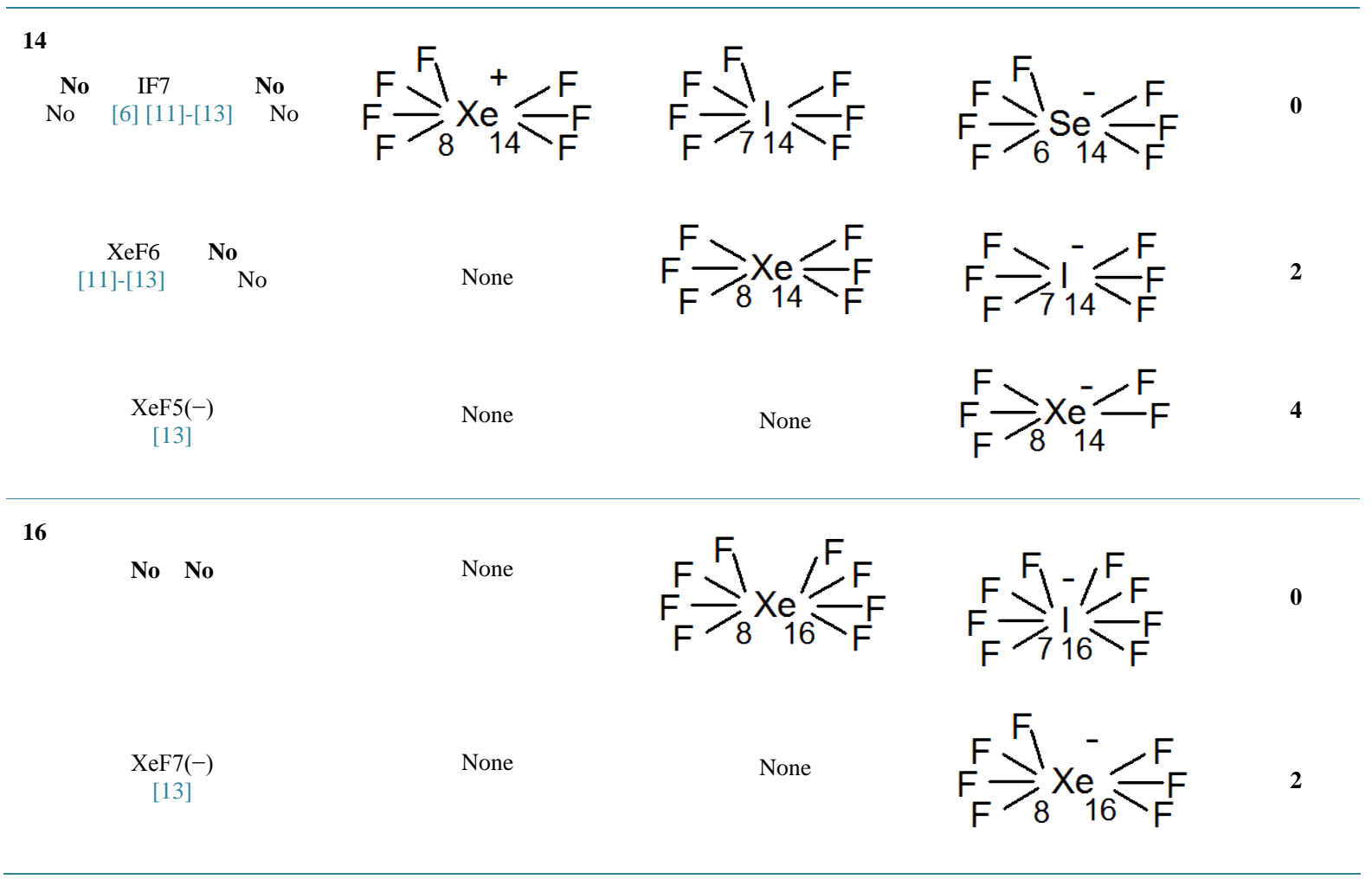

In Table 1, structural formulas of effective-valence isoelectronic compounds are methodically drawn. The central elements are from column 1 to 8 in the periodic table and every column of the periodic table is represented. External atoms are connected to the charged or uncharged central atom via one covalent bond. This is why surrounding atoms belong to column 1 or 7 in the periodic table.

In Table 1, compounds in horizontal series follow the effective-valence isoelectronicity rule. Column 1 gives the right side number of central atoms in the series and the name of each compound in the series. In one series, the left side number i.e. the valence number of the centers are different, according to the isoelectronicity rule. Compounds in each series appear in columns 2, 3 and 4 of Table 1.

Series also are arranged in groups of lines showing central atoms sharing the same Lewis number. This Lewis number is written in column 1 of Table 1 , as well as on the right side of the center atoms in column 2 to 4 . It ranges from zero for $\mathrm{Na}(+)$ up to 16 for $\mathrm{XeF7}(-)$ (on the last line).

The fifth column indicates the even number of remaining electrons in the outer-shell of the central atoms. This even number ranges from 0 for $\mathrm{Na}(+)$ in the first line of Table 1 , up to 8 for $\mathrm{F}(-)$; with special values of 0 or 8 for $\mathrm{Xe}$ as a noble gas; see $\mathrm{XeF}(-)$.

Globally, Table 1 has 59 known compounds and 14 unknowns. The large number of known compounds seems to validate both rules: The even-odd rule and the proposed definition of the effective-valence isoelectronicity rule. The existence of the unknown compounds is discussed in the following.

\section{Discussion}

\subsection{Isoelectronic Compounds and Lewis' Octet Rule}

In Table 1, only fourteen compounds, like CH4, $\mathrm{NH} 4(+)$ and $\mathrm{BH} 4(-)$, meet Lewis' octet rule. We notice that if one of these compounds is a Lewis compound, all its isoelectronic compounds will be as well. The number of these compounds is small in comparison with the 40 other known single-bonded even-odd compounds. We already know that the group of Lewis compounds is encompassed in the group of even-odd compounds; it seems thus possible that another rule exits, including Lewis' octet compounds, even-odd compounds and some more. This new group could include unpaired electrons or multi-bonded connections. 


\subsection{Similar Charge Positions between References and the Even-Odd Rule}

When comparing results of the even-odd rule to those given in scientific literature, charge position is the same for the following compounds and their isoelectronic ions: $\mathrm{BF} 3, \mathrm{SiH} 2, \mathrm{CH} 4, \mathrm{NH} 3, \mathrm{H} 2 \mathrm{O}, \mathrm{HCl}$ and PF5. The charge is systematically located on the central atom in the structure. Twenty-one compounds are concerned. It is nearly $50 \%$ of the known compounds listed in Table 1 . As a consequence, the existence of these 21 compounds in Table 1 , may be interpreted as a valid confirmation of the usefulness of the even-odd rule and of the effective-valence isoelectronic rule.

\subsection{Central Atoms without Covalent Bonds}

Several compounds in Table 1 are composed of single atoms, charged or neutral. About $50 \%$ of these monoatomic compounds following the even-odd and the isoelectronic rules are referenced in scientific literature.

Neutral elements in column 3, are: Be, C, O and Xe. Their structural formulas follow the even-odd rule. Information is available on each of them: their chemical and physical properties are known and they can remain monoatomic in the gas phase at sufficiently high temperature.

Monoatomic cations are $\mathrm{Na}(+), \mathrm{B}(+), \mathrm{N}(+)$ and $\mathrm{F}(+)$ in column 2. They are compatible with the even-odd rule. As shown on the same line, ion $\mathrm{Na}(+)$ is isoelectronic with Xenon, a noble gas. Both are known compounds. The three other cations, $\mathrm{B}(+), \mathrm{N}(+)$ and $\mathrm{F}(+)$ are not known entities. Is this due to specific chemical properties that prevent them from existing in an independent form or are they inexistent? A definitive answer can only be given when these ions are synthetized and analyzed.

Among monoatomic anions in column 4, $\mathrm{Na}(-), \mathrm{B}(-), \mathrm{N}(-), \mathrm{F}(-)$, only $\mathrm{F}(-)$ is known. Is it possible to produce and experiment on the three others? For example, $\mathrm{N}(-)$ is isoelectronic with monoatomic oxygen. As the latter is obtained at high temperature, could it be the same for $\mathrm{N}(-)$ ?

\subsection{Central Atoms with Their Highest Number of Electrons in the Outer-Shell}

The highest number of electrons in the outer-shell is displayed by xenon. Compounds of this noble gas are very particular; they are not known for other noble gas. In Table 1, about $75 \%$ of them are referenced to in scientific literature.

Among neutral molecules listed in column 3, xenon single-bonded compounds are Xe (see group 8), XeF2, $\mathrm{XeF} 4, \mathrm{XeF6}$ and XeF8. Except for XeF8, their properties are known. Is it possible to obtain information about this molecule?

Among cations in column 2, they are: $\mathrm{XeF}(+), \mathrm{XeF} 3(+), \mathrm{XeF5}(+)$ and $\mathrm{XeF7}(+)$. Only the last one is unknown. Since its isoelectronic molecule IF7 is known, is it possible to produce it?

Among anions in column 4 at last, they are: $\mathrm{XeF}(-), \mathrm{XeF} 3(-), \mathrm{XeF5}(-)$ and $\mathrm{XeF7}(-)$. Two of them are known: $\mathrm{XeF5}(-)$ and XeF7(-). An expansion of our knowledge, mainly for XeF3(-), even if it does not have an isoelectronic compounds, may be possible.

\subsection{Standard Compounds}

Among all other isoelectronic compounds obtained with both even-odd and isoelectronic rules, only three are not referenced in scientific literature. These are NaCl2(-), SeF7(-) and IF8(-). There are only three unknown compounds in comparison with the large number of well-known isoelectronic compounds (more than 40) shown in Table 1. It may be interesting to find which physical property is preventing their synthesis or their study.

\subsection{Isoelectronic Group and the Even-Odd Rule}

In Table 1, each triplet of isoelectronic compounds is drawn in agreement with the even-odd rule. In some triplets, one compound is unknown although we suppose that they can exist. An argument supporting this hypothesis is: one compound not following the even-odd rule belongs to an isoelectronic group and none of the group can be included in the even-odd rule. This argument seems acceptable even if it is in a negative form. It is therefore possible that unknown single-bonded compounds will become known compounds.

\subsection{Localized Electrical Effect of Charges}

On any line of Table 1, two isoelectronic compounds have the same covalent-bond configurations. The charge 
itself, located in the center, has therefore no impact on electrical properties of bonds. As a consequence, the electrical effect of the charge can only be localized in the electron inner-shell and on the nucleus. Scientific literature is unfortunately not consistent on that subject and many structures, drawn using classical methods, indicate a charge globally spread over the entire compound [14]. This apparent contradiction will probably be explained for multi-bonded compounds.

\section{Conclusions}

Structural formulas of several known and unknown single-bonded compounds are drawn using the even-odd rule and the effective-valence isoelectronicity rule. They are sorted by the value of the Lewis number and the number of electrons in the outer-shell. This organization forms groups of compounds and gives an independent way to test the effectiveness of the even-odd rule with the definition of the effective-valence isoelectronicity. As a consequence, isoelectronic single-bonded compounds form several groups completely included in the evenodd rule.

The validity of both rules is here confirmed for a large number of known compounds even if information is missing for about $15 \%$ of them. Each of these $15 \%$ is isoelectronic with known single-bonded compounds. These unavailable compounds like $\mathrm{N}(+), \mathrm{XeF} 4$ or $\mathrm{XeF} 3(-)$ may be under investigations to improve the number of known compounds and to confirm the global importance of both rules.

The next step to check the even-odd rule will probably be an adaptation to multiple-bonded compounds.

\section{References}

[1] Icilio, G. (1911) Amedeo Avogadro e la sua opera scientific. Accademia delle scienze, Opere scelte di Amedeo Avogadro, Turin, i-cxl.

[2] Greenaway, F. (1966) John Dalton and the Atom. Cornell University Press, Ithaca.

[3] Einstein, A. (1905) Über die von der molekularkinetischen Theorie der Wärmegeforderte Bewegung von in ruhenden Flüssigkeiten suspendierten Teilchen. Annalen der Physik, 322, 549-560.

[4] Perrin, J. (1909) Mouvement brownien et réalité moléculaire. Annales de Chimie et de Physique, 18, 1-114.

[5] Faraday, M. (1859) Experimental Researches in Chemistry and Physics. Richard Taylor and William Francis, London.

[6] Greenwood, N.N. and Earnshaw, A. (1997) Chemistry of the Elements. 2nd Edition, Butterworth-Heinemann, Oxford.

[7] DeKock, R.L. and Gray, H.B. (1989) Chemical Structure and Bonding. University Science Books, 94.

[8] http://en.wikipedia.org/wiki/Isoelectronicity

[9] Auvert, G. (2014) Improvement of the Lewis-Abegg-Octet Rule Using an "Even-Odd” Rule in Chemical Structural Formulas: Application to Hypo and Hyper-Valences of Stable Uncharged Gaseous Single-Bonded Molecules with Main Group Elements. Open Journal of Physical Chemistry, 4, 60-66. http://dx.doi.org/10.4236/ojpc.2014.42009

[10] Auvert, G. (2014) Chemical Structural Formulas of Single-Bonded Ions Using the "Even-Odd” Rule Encompassing Lewis's Octet Rule: Application to Position of Single-Charge and Electron-Pairs in Hypo- and Hyper-Valent Ions with Main Group Elements. Open Journal of Physical Chemistry, 4, 67-72. http://dx.doi.org/10.4236/ojpc.2014.42010

[11] http://www.chemspider.com/

[12] http://www.ncbi.nlm.nih.gov/

[13] http://en.wikipedia.org/

[14] Gillespie, R.J. and Popelier, P.L.A. (2001) Chemical Bonding and Molecular Geometry. Oxford University Press, Oxford. 
Scientific Research Publishing (SCIRP) is one of the largest Open Access journal publishers. It is currently publishing more than 200 open access, online, peer-reviewed journals covering a wide range of academic disciplines. SCIRP serves the worldwide academic communities and contributes to the progress and application of science with its publication.

Other selected journals from SCIRP are listed as below. Submit your manuscript to us via either submit@scirp.org or Online Submission Portal.
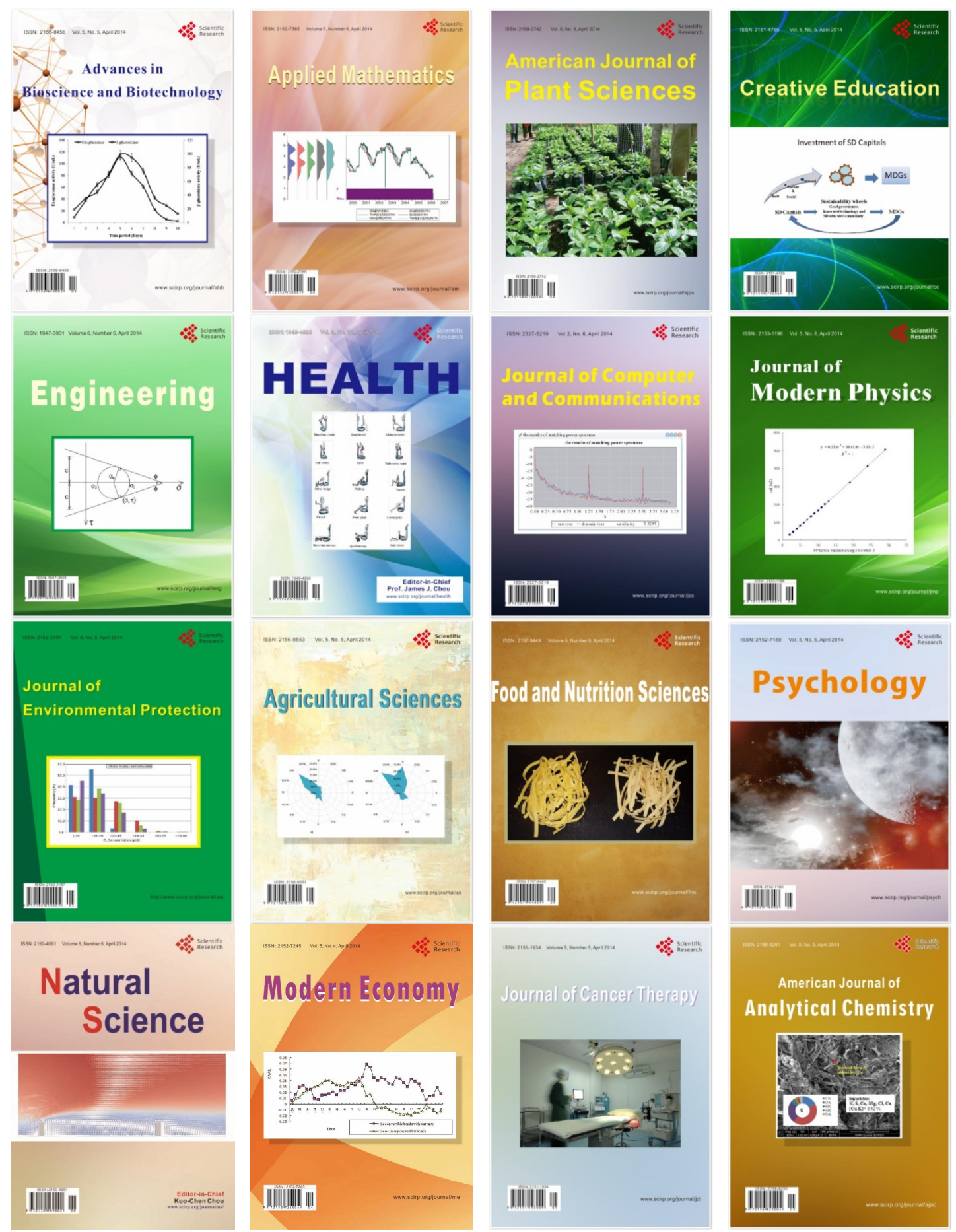